\title{
Long non-coding RNA LINC00460 predicts poor survival and promotes cell viability in pancreatic cancer
}

\author{
JUNFENG SUN, JIANYING YANG, KUI LV and JIANGUO GUAN \\ Department of Emergency, Anhui No. 2 Provincial People's Hospital, Hefei, Anhui 230041, P.R. China
}

Received September 2, 2019; Accepted April 3, 2020

DOI: 10.3892/ol.2020.11652

\begin{abstract}
Emerging evidence has demonstrated that long non-coding RNAs (lncRNAs) possess great potential as vital biomarkers and powerful therapeutic targets in various diseases. In the present study, differentially expressed transcripts in pancreatic cancer (PC) were identified, and a competing endogenous RNA (ceRNA) network was constructed using The Cancer Genome Atlas database. An independent cohort consisting of 59 patients with PC was used to validate the clinical value of the identified lncRNA. Cell viability and colony formation assays were used to evaluate the biological functions of the lncRNA in PC cells. The present bioinformatic analysis revealed that LINC00460 was upregulated in PC samples with a prognostic significance. In the ceRNA network, it potentially targeted the microRNA-503/cyclin D1 axis. The results of real-time quantitative PCR confirmed that LINC00460 was significantly upregulated in cancer tissues and was associated with poor survival of patients with PC. The expression levels of LINC00460 were significantly associated with tumor size, but not with age, sex, differentiation, lymph node metastasis, vascular invasion and tumor stage. Through univariate and multivariate analysis, LINC00460 was characterized as an independent prognostic biomarker for PC. Further in vitro experiments demonstrated that suppressing LINC00460 using small interfering RNA inhibited viability and colony formation of PC cells. In summary, LINC00460 may be an independent prognostic biomarker for $\mathrm{PC}$ and may serve as an oncogenic lncRNA that promotes PC cell growth. Further in-depth exploration is required to reveal the specific biological mechanism of LINC00460 in PC cells.
\end{abstract}

Correspondence to: Dr Jianguo Guan, Department of Emergency, Anhui No. 2 Provincial People's Hospital, 1868 Dangshan Road, Hefei, Anhui 230041, P.R. China

E-mail: guanjg1978@163.com

Key words: pancreatic cancer, LINC00460, competing endogenous RNA network, prognosis

\section{Introduction}

Pancreatic cancer (PC) is one of the most vicious malignancies with $\sim 56,770$ new cases and 45,750 deaths in the United States in 2019 (1). It is generally considered as an incurable disease, with an unsatisfactory therapeutic outcome following general treatment methods, including surgery, chemotherapy and radiotherapy (2). The 5-year survival rate of patients with PC diagnosed between 2008 and 2014 was only 9\% in the United States, representing the lowest survival rate among all cancer types according to the American Cancer Society (1). Therefore, it is urgent to investigate the molecular mechanisms underlying PC and to identify more effective therapeutic strategies and prognostic biomarkers.

The human transcriptome is composed of a considerable number of protein-coding mRNAs as well as non-coding transcripts. Long non-coding RNAs (lncRNAs) are transcripts $>200$ nucleotides in length without protein-coding ability, which possess vital roles in gene expression regulation at the epigenetic, transcriptional and post-transcriptional levels (3). MicroRNAs (miRNAs/miRs) are a series of small non-coding RNAs $<22$ nucleotides in length originating from hairpin precursors (4). miRNAs couple with the 3'-untranslated region of their target genes and decrease protein expression by inducing mRNA degradation, or by inhibiting mRNA translation (5). However, the biological and clinical roles of lncRNAs and miRNAs in PC are not thoroughly understood, and require further investigation. A common functional mechanism of lncRNAs in cancer cells is to couple with their target miRNAs, leading to silencing of downstream genes, which is generally known as the "competing endogenous RNA (ceRNA)' theory (6). Construction of a ceRNA network using differentially expressed transcripts in cancer could help to identify biomarkers or therapeutic targets for PC.

LINC00460, located on chromosome 13: 106376564-106378595, has been reported to promote the progression in numerous types of cancer, including nasopharyngeal carcinoma (7), lung cancer (8), gastric cancer (9) and esophageal squamous cell carcinoma (10). However, it remains unclear whether LINC00460 is dysregulated and serves an important role in PC tumorigenesis. In the present study, The Cancer Genome Atlas (TCGA) database was used to construct a ceRNA network containing differentially expressed lncRNAs, miRNAs and mRNAs. Furthermore, tissue samples were collected and in vitro experiments were performed to 
validate the pivotal lncRNA predicted in the ceRNA network, LINC00460.

\section{Materials and methods}

Bioinformatic analysis. The expression profiles of mRNAs and miRNAs were obtained from the TCGA-PDAC dataset (http://cancergenome.nih.gov/) (11), which contained 146 PC and 3 normal samples. Based on the lncRNA information in the GENECODE database (https://www.gencodegenes. org/), the IncRNA expression profile was extracted from the mRNA dataset. The package 'edgeR' v3.28.0 (http://www. bioconductor.org/packages/release/bioc/html/edgeR.html) of the R software v3.6.1 (https://www.r-project.org/) was utilized to screen out differentially expressed transcripts using the threshold values of $\log _{2}$ fold changel $>1$ and $\mathrm{P}<0.05$. The interactions between IncRNAs and miRNAs were predicted using miRcode (http://www.mircode.org/index.php). Three databases, including miRTarBase (http://mirtarbase.mbc. nctu.edu.tw/php/index.php), miRDB (http://mirdb.org/) and TargetScan (http://www.targetscan.org/vert_72/), were used to predict targeted mRNAs of miRNAs. All differentially expressed mRNAs, miRNAs and lncRNAs were used to construct a ceRNA network that was visualized and modified using the Cytoscape software v3.7.0 (12). The prognostic value of lncRNAs for the overall survival of patients with PC was evaluated using a log-rank test through the Gene Expression Profiling Interactive Analysis (GEPIA) website (http://gepia.cancer-pku.cn/index.html) (13).

Tissue samples. The present study was approved by the Research Ethics Committee of Anhui No. 2 Provincial People's Hospital (Hefei, China). Written informed consent was obtained from all subjects. A total of 59 patients with PC (35 males and 24 females, aged between 52 and 75 years) receiving surgical excision therapy at the aforementioned hospital, and admitted between March 2016 and December 2017, were enrolled in the present study. All patients had pathologically confirmed pancreatic ductal adenocarcinoma, and none of the patients received preoperative adjuvant chemotherapy or radiotherapy. All patients received standard gemcitabine-based postoperative adjuvant chemotherapy. The last follow-up time point was July 16, 2019. The cancer and adjacent normal tissues $(>1 \mathrm{~cm}$ from the margin of the cancer tissue) were collected from the excised surgical specimens and stored in sterile enzyme-free tubes. They were immediately frozen in liquid nitrogen and stored at $-80^{\circ} \mathrm{C}$ until subsequent experimentation. The general and clinicopathological characteristics of the patients were recorded using electronic health records.

Reverse transcription-quantitative PCR (RT-qPCR). Total RNA from cancer and normal tissues was extracted using TRIzol ${ }^{\circledR}$ reagent (Invitrogen; Thermo Fisher Scientific, Inc.). The RNA concentration and purity were measured using the NanoDrop 2000 (Thermo Fisher Scientific, Inc.). RT was performed using the PrimeScript ${ }^{\mathrm{TM}}$ RT reagent kit with gDNA Eraser (Takara Biotechnology Co., Ltd.) at $37^{\circ} \mathrm{C}$ for $15 \mathrm{~min}$ and $85^{\circ} \mathrm{C}$ for $5 \mathrm{sec}$ to obtain cDNA. The quantitative measurement of LINC00460 expression was analyzed using the Applied Biosystems 7900 system with the One-Step TB Green ${ }^{\circledR}$
PrimeScript $^{\mathrm{TM}}$ PLUS RT-PCR kit (Takara Biotechnology Co., Ltd.) with the following thermocycling conditions: $42^{\circ} \mathrm{C}$ for $5 \mathrm{~min}$ and $95^{\circ} \mathrm{C}$ for $10 \mathrm{sec}$, followed by 40 cycles of $95^{\circ} \mathrm{C}$ for $3 \mathrm{sec}$ and $60^{\circ} \mathrm{C}$ for $30 \mathrm{sec}$. The following primers were used: GAPDH forward, 5'-TCGGAGTCAACGGATTTGGT-3' and reverse, 5'-TTGGAGGGATCTCGCTCCT-3'; LINC00460 forward, 5'-ACAGCATGAGCCAGGACATC-3' and reverse, 5'-GAAAGCTGCAACATGCTCCC-3'. LINC00460 expression was normalized to that of GAPDH, and the relative LINC00460 expression level was determined using the $2^{-\Delta \Delta C q}$ method (14).

Cell culture and small interfering RNA (siRNA) transfection. The human PC PANC1 and SW1990 cell lines were purchased from Wuhan Boster Biological Technology, Ltd., and were cultured in DMEM supplemented with 10\% FBS (both Thermo Fisher Scientific, Inc.) and 1\% penicillin/streptomycin at $37^{\circ} \mathrm{C}$ with $5 \% \mathrm{CO}_{2}$. The siRNA oligos (si-LINC00460; 5'-GUGUCAACAACCUGUUUAAUU-3') and the negative control (si-NC; 5'-UUCUCCGAACGUGUCACGUTT-3') were purchased from Guangzhou RiboBio Co., Ltd. PANC1 and SW1990 cells were transfected with $100 \mathrm{nM}$ siRNA using Lipofectamine ${ }^{\circledR} 2000$ (Invitrogen; Thermo Fisher Scientific, Inc.) according to the manufacturer's protocol. Subsequent experiments were conducted $48 \mathrm{~h}$ after siRNA transfection.

Cell viability assay. A total of $1 \times 10^{3}$ cells/well were incubated for 24,48 and $72 \mathrm{~h}$ at $37^{\circ} \mathrm{C}$. At the aforementioned time points, MTT was added to the culture medium to form a $0.5 \mathrm{mg} / \mathrm{ml}$ MTT solution. The spent culture medium was discarded $4 \mathrm{~h}$ later and replaced with $100 \mu \mathrm{l}$ DMSO to dissolve the purple formazan. After mixing the plate gently for $10 \mathrm{~min}$, the formazan crystals were dissolved. Cell viability was analyzed at a wavelength of $570 \mathrm{~nm}$ using the BioTek microplate reader (Agilent Technologies, Inc.).

Colony formation assay. A total of 300 cells/well were uniformly seeded into a 6-well plate. After $24 \mathrm{~h}$, the medium was replaced and the cells were incubated with $2 \mathrm{ml}$ culture medium for a further 2 weeks. The medium was then discarded, and the cells were washed twice with PBS. The cells were stained using Giemsa stain for $30 \mathrm{~min}$ at room temperature and the colony number in each well was counted manually under a light microscope (magnification, $\mathrm{x} 4$ ).

Statistical analysis. All statistical analyses were performed using SPSS v19.0 (IBM Corp.). Data are presented as the mean $\pm S D(n=3)$. Differences in the expression levels between the cancer tissues and adjacent normal tissues were analyzed using a paired Student's t-test. For $\geq 3$ groups, one-way ANOVA followed by Fisher's least significant difference post-hoc test were performed. The $\chi^{2}$ test was used to assess the association between LINC00460 expression and the clinicopathological features of patients with PC. The Cox regression model was used for univariate and multivariate analyses, in which clinicopathological features (age, sex, differentiation, tumor size, lymph node metastasis and vascular invasion) served as covariates. Kaplan-Meier analysis was used to evaluate the prognostic value of LINC00460 expression. LINC00460 expression higher than the average was defined as high 

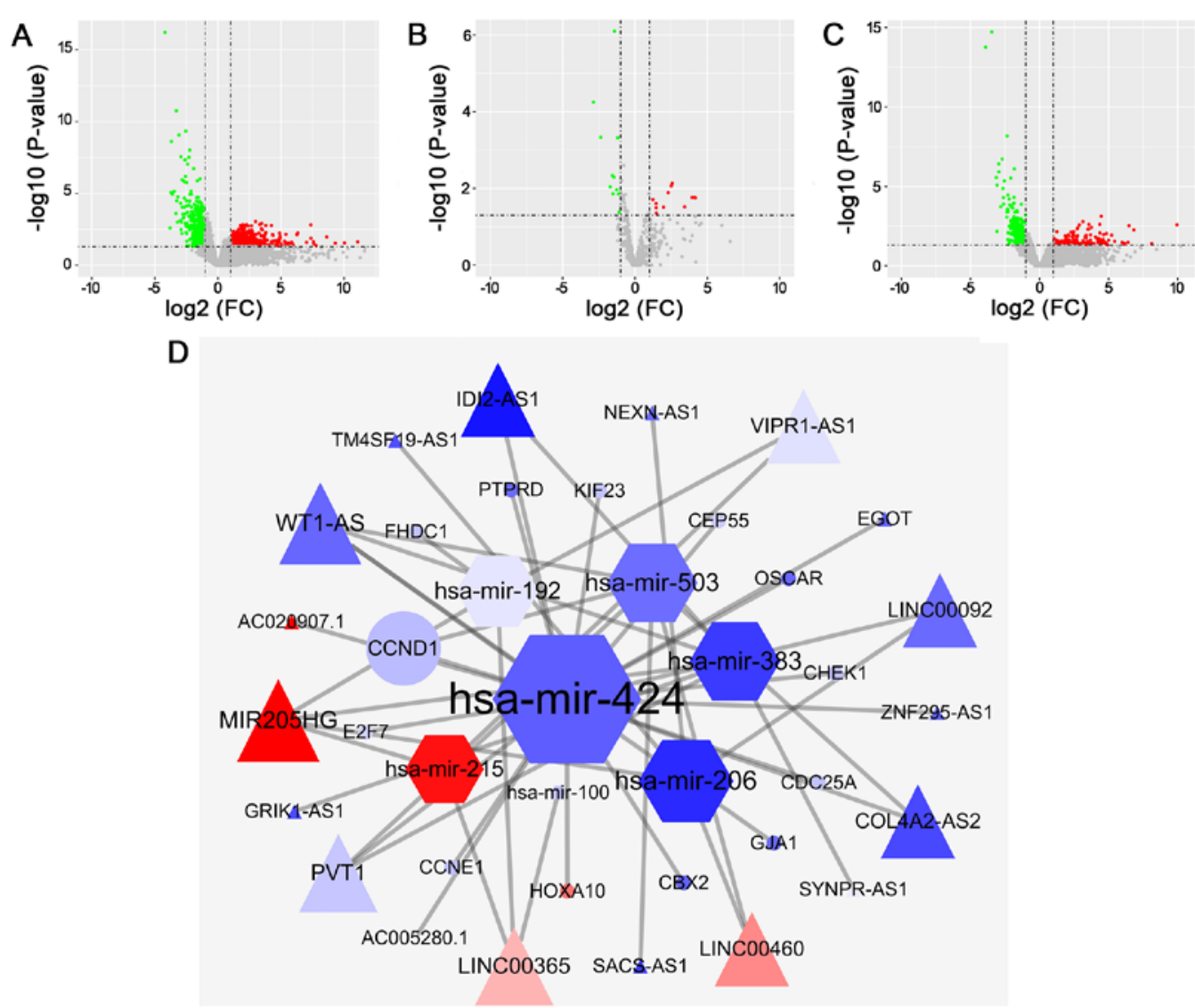

Figure I. LINC00460 is a key lncRNA in PC according to bioinformatics analysis. Volcano plots of differentially expressed (A) mRNAs, (B) miRNAs and (C) IncRNAs in PC, compared with normal tissues. (D) Competing endogenous RNA network constructed using differentially expressed mRNAs (circles), miRNAs (hexagons) and lncRNAs (triangles) based on miRcode, miRTarBase, miRDB and TargetScan. The red and blue in the network represent upregulated and downregulated transcripts, respectively. PC, pancreatic cancer; miRNA, microRNA; lncRNA, long non-coding RNA; FC, fold change.

expression, while LINC00460 expression lower than the average was defined as low expression. Pearson correlation was applied to analyze the correlation between $\mathrm{Ki}-67$ and LINC00460 expression. $\mathrm{P}<0.05$ was considered to indicate a statistically significant difference.

\section{Results}

Bioinformatic analysis identifies LINC00460 as a key lncRNA in $P C$. To identify prognostic biomarkers for $\mathrm{PC}$, an integrated bioinformatics analysis was conducted using $\mathrm{PC}$ data from the TCGA. This analysis identified differentially expressed mRNAs (476 upregulated and 436 downregulated; Fig. 1A), miRNAs (12 upregulated and 13 downregulated; Fig. 1B) and lncRNAs (142 upregulated and 218 downregulated; Fig. 1C) in cancer tissues, compared with normal tissues. miRcode identified 18 differentially expressed lncRNAs (AC005280.1, GRIK1-AS1, AC020907.1,WT1-AS,LINC00365,COL4A2-AS2,LINC00092, SACS-AS1, MIR205HG, VIPR1-AS1, IDI2-AS1, LINC00460, TM4SF19-AS1,NEXN-AS1,EGOT,ZNF295-AS1,SYNPR-AS1 and PVT1) and seven differentially expressed lncRNA-targeted miRNAs (hsa-mir-424, hsa-mir-206, hsa-mir-503, hsa-mir-192, hsa-mir-383, hsa-mir-100 and hsa-mir-215). miRTarBase, TargetScan and the miRDB database identified 13 differentially expressed miRNA-targeted mRNAs: Chromobox 2, gap junction protein alpha 1 , cell division cycle $25 \mathrm{~A}$, checkpoint kinase 1 , cyclin D1 (CCND1), osteoclast associated Ig-like receptor, centrosomal protein 55 , protein tyrosine phosphatase receptor type D, kinesin family member 23 , homeobox A10, E2F transcription factor 7, FH2 domain containing 1 and cyclin E1. Using these significantly upregulated or downregulated transcripts in PC, a ceRNA network was constructed using the Cytoscape platform (Fig. 1D). The prognostic significance of lncRNAs involved in the network was evaluated using the GEPIA platform. The results revealed that expression levels of LINC00460 (Fig. 2A), EGOT (Fig. 2B), VIPR1-AS1 (Fig. 2C) and SACS-AS1 (Fig. 2D) were significantly associated with the survival rate of patients with PC $(\mathrm{P}<0.05)$. Among these four prognosis-associated lncRNAs, the fold change of LINC00460 expression was the highest (3.47 times higher than the normal control; data not shown). Therefore, LINC00460 was selected as the target to be validated in the PC cohort enrolled in the present study.

LINC00460 is an independent prognostic biomarker for PC. To test the results of the bioinformatic analysis, LINC00460 expression was examined in 59 paired $\mathrm{PC}$ and normal tissues. The expression levels of LINC00460 quantified by RT-qPCR were significantly upregulated in cancer tissues compared with those in normal tissues $(\mathrm{P}<0.001$; Fig. 3A). Additionally, the Kaplan-Meier plot revealed that high LINC00460 expression predicted worse survival of patients with PC compared with low expression $(\mathrm{P}<0.05$; Fig. $3 \mathrm{~B})$, which was consistent with the predicted result from GEPIA. LINC00460 expression and clinical parameters of patients are summarized in Table I. 

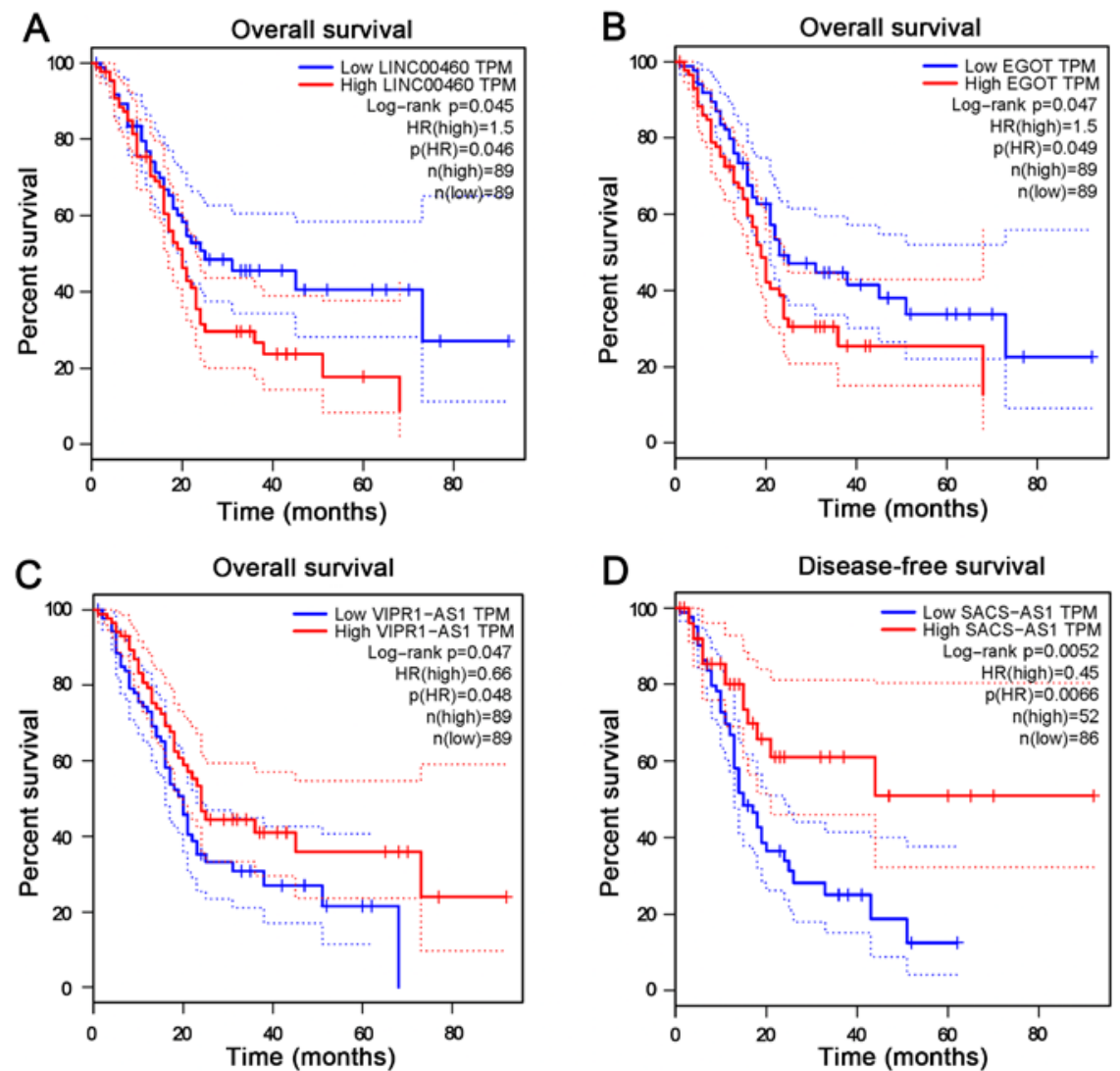

Figure 2. Prognostic value of differentially expressed long non-coding RNAs predicted using the GEPIA website. (A) High expression levels of LINC00460 predicted poor OS of patients with PC. (B) High EGOT expression was significantly associated with poor OS in patients with PC. (C) High VIPR1-AS1 expression was associated with good OS of patients with PC. (D) High SACS-AS1 expression predicted good disease-free survival of patients with PC. Expression levels higher than the average were defined as high expression, while expression levels lower than the average were defined as low expression. PC, pancreatic cancer; OS, overall survival; GEPIA, Gene Expression Profiling Interactive Analysis; HR, hazard ratio; TPM, transcripts per million.
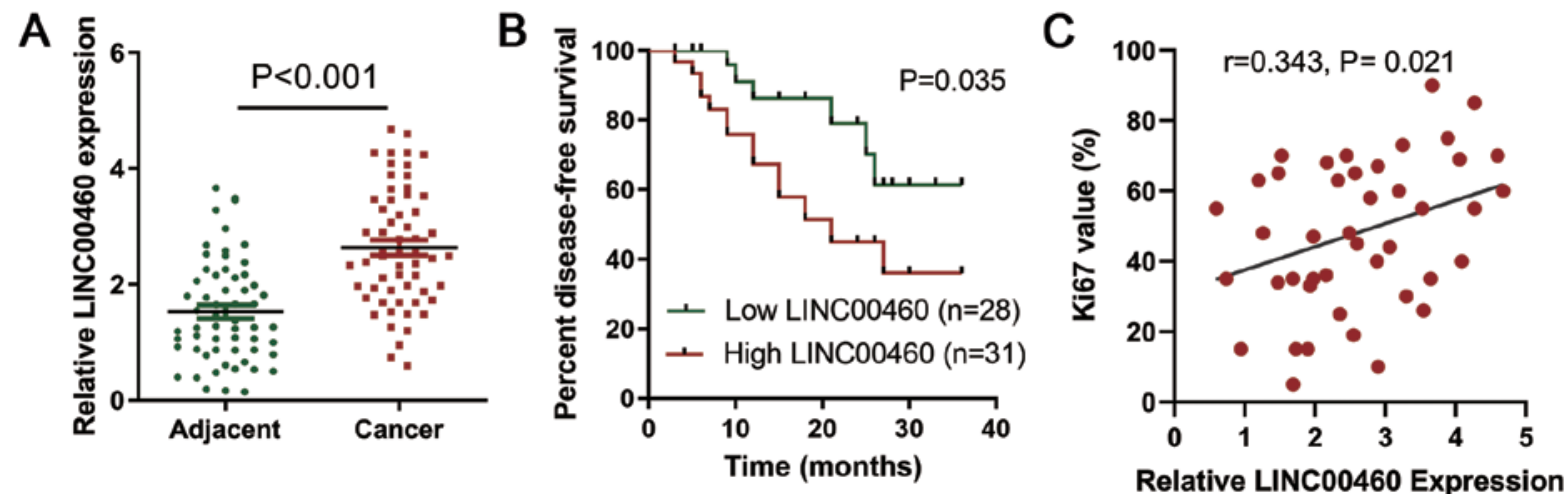

Figure 3. LINC00460 expression in PC tissues and its prognostic significance in 59 patients with PC. (A) Expression levels of LINC00460 were significantly increased in cancer tissues compared with those in adjacent normal tissues $(\mathrm{P}<0.001)$. (B) High LINC00460 expression predicted a shorter overall survival of patients with PC compared with low LINC00460 expression. (C) High LINC00460 expression was significantly associated with high Ki67 values in PC tissues $(\mathrm{r}=0.343 ; \mathrm{P}<0.05)$. LINC00460 expression higher than the average was defined as high expression, while LINC00460 expression lower than the average was defined as low expression. PC, pancreatic cancer.

The expression levels of LINC00460 were statistically associated with tumor size $(\mathrm{P}<0.05)$, but not with age, sex, differentiation, vascular invasion, lymph node metastasis and tumor stage. The univariate analysis revealed that lymph node metastasis [hazard ratio (HR), 0.721; 95\% CI, 0.545-0.898; $\mathrm{P}<0.01]$, tumor size $(\mathrm{HR}, 0.640 ; 95 \% \mathrm{CI}, 0.442-0.925 ; \mathrm{P}<0.01)$ and expression levels of LINC00460 (HR, 0.698; 95\% CI, $0.433-0.916 ; \mathrm{P}<0.05)$ were prognostic parameters for $\mathrm{PC}$.
Furthermore, multivariate analysis confirmed that LINC00460 expression (HR, 0.751; 95\% CI, 0.518-0.993; $\mathrm{P}<0.05$ ), tumor size $(\mathrm{HR}, 0.724 ; 95 \% \mathrm{CI}, 0.479-0.962 ; \mathrm{P}<0.05)$ and lymph node metastasis (HR, 0.848; 95\% CI, 0.663-0.975; P<0.05) were independent prognostic biomarkers for PC (Table II).

Silencing LINC00460 inhibits PC cell proliferation. The ceRNA network demonstrated that LINC00460 may act as a 
Table I. Association between LINC00460 expression and clinical parameters of 59 patients with pancreatic cancer.

\begin{tabular}{lccc}
\hline & \multicolumn{2}{c}{ LINC00460, $\mathrm{n}$} & \\
\cline { 2 - 3 } & $\begin{array}{c}\text { Low } \\
\text { Parameters }\end{array}$ & $\begin{array}{c}\text { High } \\
\text { expression }\end{array}$ & P-value \\
\hline Age, years & & & 0.358 \\
$\leq 60$ & 12 & 17 & \\
$>60$ & 16 & 14 & \\
Sex & & & 0.205 \\
Male & 19 & 16 & \\
Female & 9 & 15 & \\
Differentiation & & & \\
Well/moderate & 18 & 14 & 0.141 \\
Poor & 10 & 17 & \\
Tumor size, cm & & & $0.028^{\mathrm{a}}$ \\
$\leq 4$ & 17 & 10 & \\
$>4$ & 11 & 21 & \\
Lymph node metastasis & & & 0.500 \\
N0 & 11 & 14 & \\
N1 & 19 & 17 & \\
Vascular invasion & & & 0.253 \\
No & 15 & 12 & \\
Yes & 13 & 19 & \\
Stage & & & \\
I-IIA & 11 & 14 & 0.500 \\
IIB-IV & 19 & 17 & \\
\hline
\end{tabular}

${ }^{a} \mathrm{P}<0.05$. LINC00460 expression higher than the average was defined as high expression, while LINC00460 expression lower than the average was defined as low expression.

molecular sponge of miR-503 and further regulate CCND1, which has been recognized as an oncogene in different types of cancer $(15,16)$. Therefore, it was hypothesized that LINC00460 may regulate PC cell proliferation. In PC tissues, high expression levels of LINC00460 were significantly associated with high Ki67 expression ( $r=0.343 ; \mathrm{P}<0.05$; Fig. 3C), which reflected the proliferative potential of cells. siRNA transfection significantly suppressed LINC00460 expression in PANC-1 and SW1990 cells (Fig. 4A). The viability of PANC-1 and SW1990 cells in the si-LINC00460 groups was significantly decreased compared with that in the si-NC and blank groups at 48 and $72 \mathrm{~h}$ (Fig. 4B and C). As shown in Fig. 4D, silencing LINC00460 expression notably reduced colony numbers, with statistical significance identified compared with the si-NC and blank groups (Fig. 4E). These in vitro results suggest that LINC00460 may have oncogenic ability and may regulate the proliferation of PC cells.

\section{Discussion}

Exploring specific biomarkers to improve survival prediction and therapeutic strategies for PC is an urgent requirement.
Accumulating evidence has demonstrated that lncRNAs regulate various critical biological functions, including transcription (17), RNA processing (18) and chromatin organization (19). Additionally, lncRNAs exert vital functions in the modulation of a large number of genes that regulate cancer cell proliferation, invasion, apoptosis and stemness $(20,21)$. Exploring key lncRNAs associated with pathological parameters and the survival of patients with PC may help to identify prognostic biomarkers and vital molecules involved in tumorigenesis. In the present study, a regulatory network of differentially expressed miRNAs, mRNAs and lncRNAs was constructed, which revealed potential molecular mechanisms, as well as helped to identify pivotal biomarkers for PC.

The clinical and biological roles of LINC00460, which is located on the human chromosome 13q33.2, have been validated in several types of cancer. Zhang et al (22) demonstrated that LINC00460 expression is notably increased in colorectal cancer and is significantly associated with clinicopathological parameters and a shorter patient survival time; additionally, they demonstrated that LINC00460 targets the miR-939-5p/LIM domain kinase 2 axis to facilitate colorectal cancer cell progression. In glioma, LINC00460 has been demonstrated to be overexpressed in cancer tissues and cell lines, and to promote cancer cell progression by negatively regulating miR-320a (23). Additionally, a recent study reported that LINC00460 could promote breast cancer cell migration, invasion and proliferation by targeting the miR-489-5p/fibroblast growth factor 7/AKT axis (24). Notably, nicotine has been found to facilitate carcinogenesis in the bronchus by activating LINC00460 and the PI3K/AKT signaling pathway (25). In the present study, the prognostic value of LINC00460 in PC was first predicted using the TCGA database and was subsequently examined in a cohort of patients with PC. It was demonstrated that high expression levels of LINC00460 were associated with poor overall survival and that LINC00460 was an independent prognostic biomarker for patients with PC.

It has been reported that the binding of miRNAs to lncRNAs is able to decrease miRNA levels, resulting in the overexpression of miRNA-targeted genes (26). The ceRNA PC network constructed in the present study may help to comprehensively understand the regulatory association between differentially expressed mRNAs and non-coding RNAs. Bioinformatics prediction revealed that in PC, LINC00460 was likely to couple with miR-503 to further regulate CCND1, a well-recognized oncogene that regulates the cell cycle and cellular proliferation $(27,28)$. This prediction is in accordance with the in vitro cell experiments of the present study, which indicated that LINC00460 modulated cell viability in PANC-1 cells.

However, the current study presents several limitations. Firstly, pathological confirmation of the non-cancerous tissues was not performed, which may have led to inaccurate expression levels of LINC00460. Secondly, the bioinformatics prediction of the ceRNA network was not confirmed by in vitro experiments. Thirdly, LINC00460 expression was only evaluated in association with disease-free survival, and no follow-up of the enrolled patients was conducted for overall survival analysis. 
Table II. Univariate and multivariate analysis identifying prognostic biomarkers for pancreatic cancer.

\begin{tabular}{|c|c|c|c|c|c|c|}
\hline \multirow[b]{2}{*}{ Parameters } & \multicolumn{3}{|c|}{ Univariate analysis } & \multicolumn{3}{|c|}{ Multivariate analysis } \\
\hline & HR & $95 \% \mathrm{CI}$ & P-value & HR & $95 \%$ CI & P-value \\
\hline Age, years ( $\leq 60$ vs. $>60)$ & 0.893 & $0.668-1.314$ & 0.452 & & & \\
\hline Sex (male vs. female) & 1.371 & $0.875-1.585$ & 0.527 & & & \\
\hline Differentiation (well/moderate vs. poor) & 1.065 & $0.881-1.242$ & 0.468 & & & \\
\hline Tumor size, cm ( $\leq 4$ vs. $>4)$ & 0.640 & $0.442-0.925$ & 0.007 & 0.724 & $0.479-0.962$ & 0.012 \\
\hline Lymph node metastasis (N0 vs. N1) & 0.721 & $0.545-0.898$ & 0.005 & 0.848 & $0.663-0.975$ & 0.020 \\
\hline Vascular invasion (no vs. yes) & 0.855 & $0.710-1.245$ & 0.496 & & & \\
\hline LINC00460 expression (low vs. high) & 0.698 & $0.433-0.916$ & 0.022 & 0.751 & $0.518-0.993$ & 0.039 \\
\hline
\end{tabular}

HR, hazard ratio.

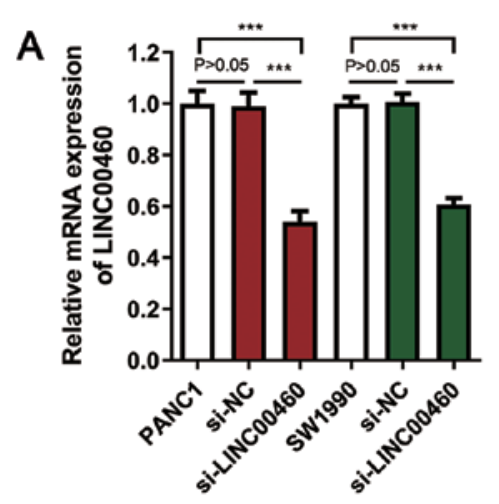

D

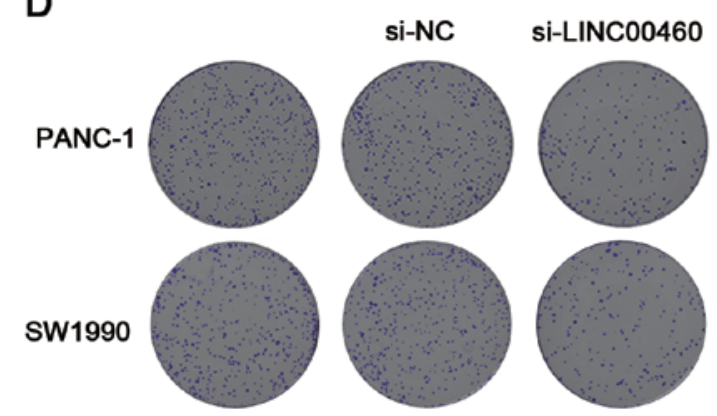

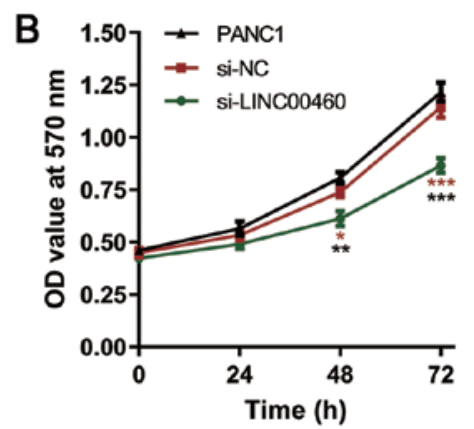

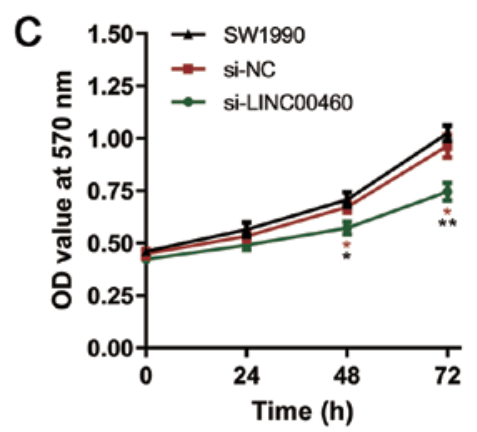

E

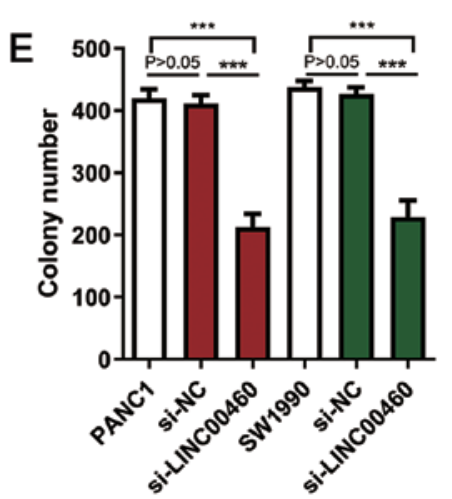

Figure 4. LINC00460 knockdown reduces the proliferative capacity of PANC-1 and SW1990 cells. (A) siRNA knockdown significantly decreased the expression levels of LINC00460 in PANC-1 and SW1990 cells. MTT assay revealing that suppressing LINC00460 inhibited the proliferation of (B) PANC-1 cells and (C) SW1990 cells at 48 and 72 h. (D) Representative images of cell colony formation of PANC-1 and SW1990 cells transfected with si-NC or si-LINC00460 (E) Colony numbers of the cells in the si-LINC00460 group were significantly lower than those in the si-NC group. ${ }^{*} \mathrm{P}<0.05$; ${ }^{* *} \mathrm{P}<0.01$; ${ }^{* * *} \mathrm{P}<0.001$. si, small interfering RNA; NC, negative control; OD, optical density.

In summary, the present study identified LINC00460 as an independent prognostic biomarker for PC and suggests that it may serve as an oncogenic lncRNA that promotes PC cell proliferation. Further in depth exploration is required to reveal the specific mechanism of LINC00460 in PC cells.

\section{Acknowledgements}

Not applicable.

\section{Funding}

No funding was received.

\section{Availability of data and materials}

The datasets used and/or analyzed during the current study are available from the corresponding author on reasonable request.

\section{Authors' contributions}

JS performed the bioinformatics analysis and wrote the manuscript. JY and KL helped to collect the data and performed the in vitro experiments. JG designed the study and drafted and revised the manuscript. All authors read and approved the final manuscript. 


\section{Ethics approval and consent to participate}

The present study was approved by the Research Ethics Committee of Anhui No. 2 Provincial People's Hospital (Hefei, China). Written informed consent was obtained from all subjects.

\section{Patient consent for publication}

Not applicable.

\section{Competing interests}

The authors declare that they have no competing interests.

\section{References}

1. DeSantis CE, Miller KD, Goding Sauer A, Jemal A and Siegel RL: Cancer statistics for African Americans, 2019. CA Cancer J Clin 69: 211-233, 2019

2. Chiorean EG and Coveler AL: Pancreatic cancer: Optimizing treatment options, new, and emerging targeted therapies. Drug Des Devel Ther 9: 3529-3545, 2015.

3. Peng WX, Koirala P and Mo YY: LncRNA-mediated regulation of cell signaling in cancer. Oncogene 36: 5661-5667, 2017.

4. Rupaimoole R and Slack FJ: MicroRNA therapeutics: Towards a new era for the management of cancer and other diseases. Nat Rev Drug Discov 16: 203-222, 2017.

5. Mansoori B, Mohammadi A, Shirjang S and Baradaran B MicroRNAs in the diagnosis and treatment of cancer. Immunol Invest 46: 880-897, 2017

6. Qi X, Zhang DH, Wu N, Xiao JH, Wang X and Ma W: ceRNA in cancer: Possible functions and clinical implications. J Med Genet 52: 710-718, 2015.

7. Kong YG, Cui M, Chen SM, Xu Y, Xu Y and Tao ZZ: LncRNA-LINC00460 facilitates nasopharyngeal carcinoma tumorigenesis through sponging miR-149-5p to up-regulate IL6 Gene 639: 77-84, 2018

8. Li K, Sun D, Gou Q, Ke X, Gong Y, Zuo Y, Zhou JK, Guo C, Xia Z, Liu L, et al: Long non-coding RNA linc00460 promotes epithelial-mesenchymal transition and cell migration in lung cancer cells. Cancer Lett 420: 80-90, 2018.

9. Wang F, Liang S, Liu X, Han L, Wang J and Du Q: LINC00460 modulates KDM2A to promote cell proliferation and migration by targeting miR-342-3p in gastric cancer. OncoTargets Ther 11: 6383-6394, 2018.

10. Liang Y, Wu Y, Chen X, Zhang S, Wang K, Guan X, Yang K, Li J and Bai Y: A novel long noncoding RNA linc00460 up-regulated by $\mathrm{CBP} / \mathrm{P} 300$ promotes carcinogenesis in esophageal squamous cell carcinoma. Biosci Rep 37: BSR20171019, 2017.

11. Cancer Genome Atlas Research Network. Electronic address: andrew_aguirre@dfci.harvard.edu; CancerGenome Atlas Research Network: Integrated genomic characterization of pancreatic ductal adenocarcinoma. Cancer Cell 32: 185-203.e13, 2017.
12. Shannon P, Markiel A, Ozier O, Baliga NS, Wang JT, Ramage D, Amin N, Schwikowski B and Ideker T: Cytoscape: A software environment for integrated models of biomolecular interaction networks. Genome Res 13: 2498-2504, 2003.

13. Tang Z, Li C, Kang B, Gao G, Li C and Zhang Z: GEPIA: A web server for cancer and normal gene expression profiling and interactive analyses. Nucleic Acids Res 45: W98-W102, 2017.

14. Livak KJ and Schmittgen TD: Analysis of relative gene expression data using real-time quantitative PCR and the 2(- $\Delta \Delta \mathrm{C}(\mathrm{T}))$ method. Methods 25: 402-408, 2001.

15. Ai B, Kong X, Wang X, Zhang K, Yang X, Zhai J, Gao R, Qi Y, Wang J, Wang Z, et al: LINC01355 suppresses breast cancer growth through FOXO3-mediated transcriptional repression of CCND1. Cell Death Dis 10: 502, 2019.

16. Qie S and Diehl JA: Cyclin D1, cancer progression, and opportunities in cancer treatment. J Mol Med (Berl) 94: 1313-1326, 2016.

17. Dykes IM and Emanueli C: Transcriptional and post-transcriptional gene regulation by long non-coding RNA. Genomics Proteomics Bioinformatics 15: 177-186, 2017.

18. Quinn JJ and Chang HY: Unique features of long non-coding RNA biogenesis and function. Nat Rev Genet 17: 47-62, 2016.

19. Han P and Chang CP: Long non-coding RNA and chromatin remodeling. RNA Biol 12: 1094-1098, 2015.

20. Schmitt AM and Chang HY: Long Noncoding RNAs in cancer pathways. Cancer Cell 29: 452-463, 2016.

21. Flynn RA and Chang HY: Long noncoding RNAs in cell-fate programming and reprogramming. Cell Stem Cell 14: 752-761, 2014.

22. Zhang Y, Liu X, Li Q and Zhang Y: IncRNA LINC00460 promoted colorectal cancer cells metastasis via miR-939-5p sponging. Cancer Manag Res 11: 1779-1789, 2019.

23. Feng L, Rao M, Zhou Y, Zhang Y and Zhu Y: Long noncoding RNA 00460 (LINC00460) promotes glioma progression by negatively regulating miR-320a. J Cell Biochem 120: 9556-9563, 2019.

24. Zhu Y, Yang L, Chong QY, Yan H, Zhang W, Qian W, Tan S, Wu Z, Lobie PE and Zhu T: Long noncoding RNA Linc00460 promotes breast cancer progression by regulating the miR-489-5p/FGF7/ AKT axis. Cancer Manag Res 11: 5983-6001, 2019.

25. Zhao H, Wang Y and Ren X: Nicotine promotes the development of non-small cell lung cancer through activating LINC00460 and PI3K/Akt signaling. Biosci Rep 39: BSR20182443, 2019.

26. Awan HM, Shah A, Rashid F and Shan G: Primate-specific Long Non-coding RNAs and MicroRNAs. Genomics Proteomics Bioinformatics 15: 187-195, 2017.

27. Huang R, Lin JY and Chi YJ: MiR-519d reduces the 5-fluorouracil resistance in colorectal cancer cells by down-regulating the expression of CCND1. Eur Rev Med Pharmacol Sci 22: 2869-2875, 2018

28. Zhu Y, Wen X and Zhao P: MicroRNA-365 inhibits cell growth and promotes apoptosis in melanoma by targeting BCL2 and Cyclin D1 (CCND1). Med Sci Monit 24: 3679-3692, 2018.

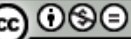

This work is licensed under a Creative Commons Attribution-NonCommercial-NoDerivatives 4.0 International (CC BY-NC-ND 4.0) License. 